\title{
Natural APOBEC3C variants can elicit differential HIV-1 restriction activity
}

\author{
Brett D. Anderson ${ }^{1 \dagger}$, Terumasa Ikeda ${ }^{1,2 \dagger}$, Seyed Arad Moghadasi ${ }^{1 \dagger}$, Amber St. Martin' ${ }^{1}$, William L. Brown ${ }^{1}$ \\ and Reuben S. Harris ${ }^{1,2^{*}}$
}

\begin{abstract}
Background: The APOBEC3 (A3) family of DNA cytosine deaminases provides an innate barrier to infection by retroviruses including HIV-1. A total of five enzymes, A3C, A3D, A3F, A3G and A3H, are degraded by the viral accessory protein Vif and expressed at high levels in CD4+ T cells, the primary reservoir for HIV-1 replication in vivo. Apart from A3C, all of these enzymes mediate restriction of Vif-deficient HIV-1. However, a rare variant of human A3C (Ile188) was shown recently to restrict Vif-deficient HIV-1 in a 293T-based single cycle infection system. The potential activity of this naturally occurring $\mathrm{A} 3 \mathrm{C}$ variant has yet to be characterized in a T cell-based spreading infection system. Here we employ a combination of Cas9/gRNA disruption and transient and stable protein expression to assess the roles of major Ser188 and minor lle188 A3C variants in HIV-1 restriction in T cell lines.

Results: Cas9-mediated mutation of endogenous A3C in the non-permissive CEM2n T cell line did not alter HIV-1 replication kinetics, and complementation with A3C-Ser188 or A3C-lle188 was similarly aphenotypic. Stable expression of A3C-Ser188 in the permissive T cell line SupT11 also had little effect. However, stable expression of A3C-Ile188 in SupT11 cells inhibited Vif-deficient virus replication and inflicted G-to-A mutations.

Conclusions: A3C-Ile188 is capable of inhibiting Vif-deficient HIV-1 replication in T cells. Although A3C is eclipsed by the dominant anti-viral activities of other A3s in non-permissive $T$ cell lines and primary $T$ lymphocytes, this enzyme may still be able to contribute to HIV-1 diversification in vivo. Our results highlight the functional redundancy in the human A3 family with regards to HIV-1 restriction and the need to consider naturally occurring variants.
\end{abstract}

Keywords: APOBEC3C, DNA cytosine deaminase, HIV-1, Human genetic variation, Innate immunity, Retrovirus restriction factor

\section{Background}

Retroviruses, including the AIDS virus human immunodeficiency virus type-1 (HIV-1), must evade destruction by an extensive array of antiviral host proteins known as restriction factors [1-3]. The seven human APOBEC3 (A3) enzymes constitute an important arm of this innate network of restriction factors. These enzymes catalyze the deamination of cytosine to uracil in single-stranded (ss) DNA substrates, and APOBEC3D (A3D), APOBEC3F

\footnotetext{
*Correspondence: rsh@umn.edu

${ }^{\dagger}$ Brett D. Anderson, Terumasa Ikeda and Seyed Arad Moghadasi have contributed equally to this work

${ }^{2}$ Howard Hughes Medical Institute, University of Minnesota, 2231 6th St.

S.E., Minneapolis, MN 55455, USA

Full list of author information is available at the end of the article
}

(A3F), APOBEC3G (A3G) and APOBEC3H (A3H) are known to contribute to HIV-1 restriction (reviewed by $[4,5])$. These four enzymes package into budding virions and, following virus entry into a new target cell, catalyze the formation of uracil lesions in reverse-transcription intermediates. These uracils template the incorporation of adenines during synthesis of the viral genomic strand, resulting in G-to-A mutations.

HIV-1 and related lentiviruses avoid lethal levels of A3 induced mutation through the virion infectivity factor (Vif), which is a small viral protein required for virus infectivity in most cell types. Vif functions as a molecular adapter to recruit $\mathrm{A} 3$ enzymes to a host ubiquitin ligase comprised of CBF- $\beta$, CUL5, RBX2, ELOB, and ELOC for polyubiquitination and subsequent degradation by 
the proteasome [6-8]. HIV-1 Vif effectively triggers the degradation of human A3D, A3F, A3G and A3H, which would otherwise restrict virus replication [9]. Through CBF- $\beta$ recruitment, Vif also downregulates the transcriptional activity of these $A 3$ genes and thereby contributes to a permissive environment for virus replication [10].

Curiously, although human APOBEC3C (A3C) elicits little Vif-deficient HIV-1 restriction activity, Vif efficiently targets it for degradation [9, 11-13]. Human A3C also is highly expressed in the primary cellular reservoir for HIV-1 replication, CD4+ T cells, and is upregulated upon HIV-1 infection similar to the other restrictive A3 proteins [9]. Moreover, human A3C also potently restricts a strain of simian immunodeficiency virus (SIV) isolated from African Green Monkeys (SIVagm) in the absence of Vif in 293T-based single cycle infection experiments [11, 14]. These observations combine to indicate that human $\mathrm{A} 3 \mathrm{C}$ is a bona fide retrovirus restriction enzyme and, further, that HIV-1 (or a SIV precursor from chimpanzees) may have recently evolved a Vif-independent mechanism for evading restriction by this enzyme (since little restriction is observed in the absence of Vif).

The $A 3$ gene family exhibits significant variation within the human population [15]. First, a deletion spanning the entire $A 3 B$ coding sequence occurs at $37 \%$ frequency worldwide with clear geographic biases $[16,17]$. Second, $A 3 H$ has at least 7 distinct haplotypes that exhibit drastically different expression levels and HIV-1 restriction phenotypes [17-21]. The most common A3H haplotype (hap I) is a poorly expressed protein with little to no Vif-deficient HIV-1 restriction activity, and it is found at a $48 \%$ frequency worldwide and implicated in cancer mutagenesis [17-22]. In contrast, the next most common A3H haplotype (hap II) is well expressed and shows strong Vif-deficient HIV-1 restriction activity [17-22]. Third, two recent reports have characterized a rare A3C-Ile188 variant ( 2\% global allele frequency) with enhanced restriction activity against Vif-deficient HIV-1 in a 293T based single cycle infection model in comparison to the predominant A3C-Ser188 enzyme $[11,23]$. Biochemical studies have indicated that the enhanced restriction activity of A3C-Ile188 may be due to increased enzyme processivity for cytosine deamination and/or to an increased propensity to homodimerize $[11,23]$.

The impact of the rare A3C-Ile188 enzyme on HIV-1 restriction in $\mathrm{T}$ lymphocytes has yet to be studied. Here, using a combination of Cas9/gRNA and stable expression approaches in $\mathrm{T}$ cell lines, we show that neither genetic disruption of $A 3 C$ in the non-permissive CEM2n T cell line, nor complementation with natural $\mathrm{A} 3 \mathrm{C}$ variants is sufficient to alter HIV-1 replication kinetics in the presence or absence of Vif. However, stable expression of
A3C-Ile188 can slow Vif-deficient virus replication and inflict G-to-A mutations in the permissive SupT11 T cell line in the absence of other A3 proteins. Taken together, these findings confirm prior reports identifying the rare Ile188 allele as a more restrictive form of A3C. Our studies underscore the possibility that this naturally occurring $\mathrm{A} 3 \mathrm{C}$ variation may influence $\mathrm{HIV}-1$ genetic variation and pathogenesis in vivo.

\section{Results}

\section{Immunoblot comparisons of epitope-tagged} and untagged $A 3 C$ variants

The first study comparing A3C-Ser188 and A3C-Ile188 used a C-terminal HA tag and reported lower expression levels of the C-terminally tagged Ile188 variant [15]. A more recent report using C-terminally HA-tagged $\mathrm{A} 3 \mathrm{C}$ variants in a different vector showed no significant expression difference [11]. Our results using different C-terminally triple-HA-tagged variants mirror the original report with the Ile188 variant expressing at lower levels (Fig. 1, top blot, lanes $2 / 3$ vs $4 / 5$ ). Another recent study used $\mathrm{N}$-terminally HA-tagged variants and found no expression difference [23]. Our results with different $\mathrm{N}$-terminally triple-HA-tagged A3C variants confirm this observation (Fig. 1, top blot, lanes 6/7 vs $8 / 9$ ). We postulate that the observed instability of the Ile 188 variant may be due to an interaction with the C-terminal HA-epitope tag in some, but not all, vectors (i.e., a potential epitope tag-associated artifact).

In an attempt to resolve this issue, we tested all available commercial anti-A3C antibodies in immunoblot experiments and found only one reagent that

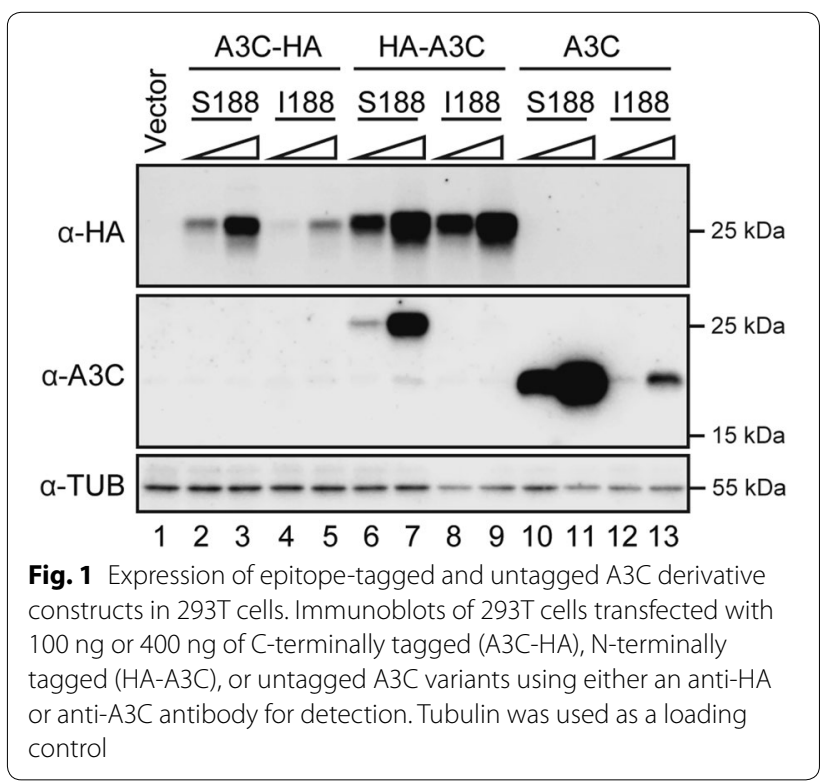


reproducibly detected both transfected and endogenous A3C (Proteintech \#10591-1-AP; see validation in Cas9/gRNA disruption studies below and "Methods" for a list of reagents that failed). This polyclonal antibody was raised against full-length $\mathrm{A} 3 \mathrm{C}$, and it enabled confirmation of a subset of the anti-epitope blots above and yielded an additional new consideration. First, it showed that the N-terminally HA-tagged A3C-Ser188 construct expresses much better than the reciprocal C-terminally HA-tagged construct (Fig. 1, middle blot, lanes $6 / 7$ vs $2 / 3$ ). Second, it strongly detected HA-A3CSer188 but only weakly the Ile188 variant (Fig. 1, middle blot, lanes $6 / 7$ vs $8 / 9$ ). This expression difference was particularly clear for untagged A3C proteins (Fig. 1, middle blot, lanes $10 / 11$ vs $12 / 13$ ). Because these proteins only differ by one amino acid, these data strongly indicate that the dominant antibody in the polyclonal mixture recognizes an epitope that requires Ser188 and is somehow compromised by Ile188. Consistent with this interpretation, a different single amino acid substitution, Ser188 to Leu, also renders A3C undetectable in immunoblot experiments with this antibody (data not shown). These epitope tag and immunoblot limitations impose significant constraints on studies with A3C.

\section{Single cycle infectivity results using 293T cells}

Because untagged, and to a lesser degree N-terminally HA-tagged, A3C-Ser188 displays visibly higher expression levels than C-terminally HA-tagged derivatives, untagged and $\mathrm{N}$-terminally HA-tagged constructs were used for functional studies from here onward. In agreement with prior reports [11, 23], untagged A3C-Ser188 caused a modest twofold reduction in the infectivity of Vif-deficient HIV-1 in the 293T-based single cycle infection system (Fig. 2a). A comparison of this A3C-Ser188 construct and a Glu68-to-Gln derivative indicated that effect is largely independent of the conserved catalytic glutamate. In contrast, A3C-Ile188 caused a larger fourfold infectivity decline that partly required deaminase activity (Fig. 2a). Parallel reactions included A3G as a strong positive control and an empty expression vector as a negative control (Fig. 2a). In comparison, similar restriction activities were observed using $\mathrm{N}$-terminally tagged A3C Ser188 and Ile188 expression constructs with a trend toward greater restriction by the Ile188 protein at higher expression levels (Fig. 2b). The untagged and N-terminally tagged constructs were not analyzed in the same experiments in parallel so head-to-head comparisons of restriction activity are not possible. The most important point from these single cycle infectivity
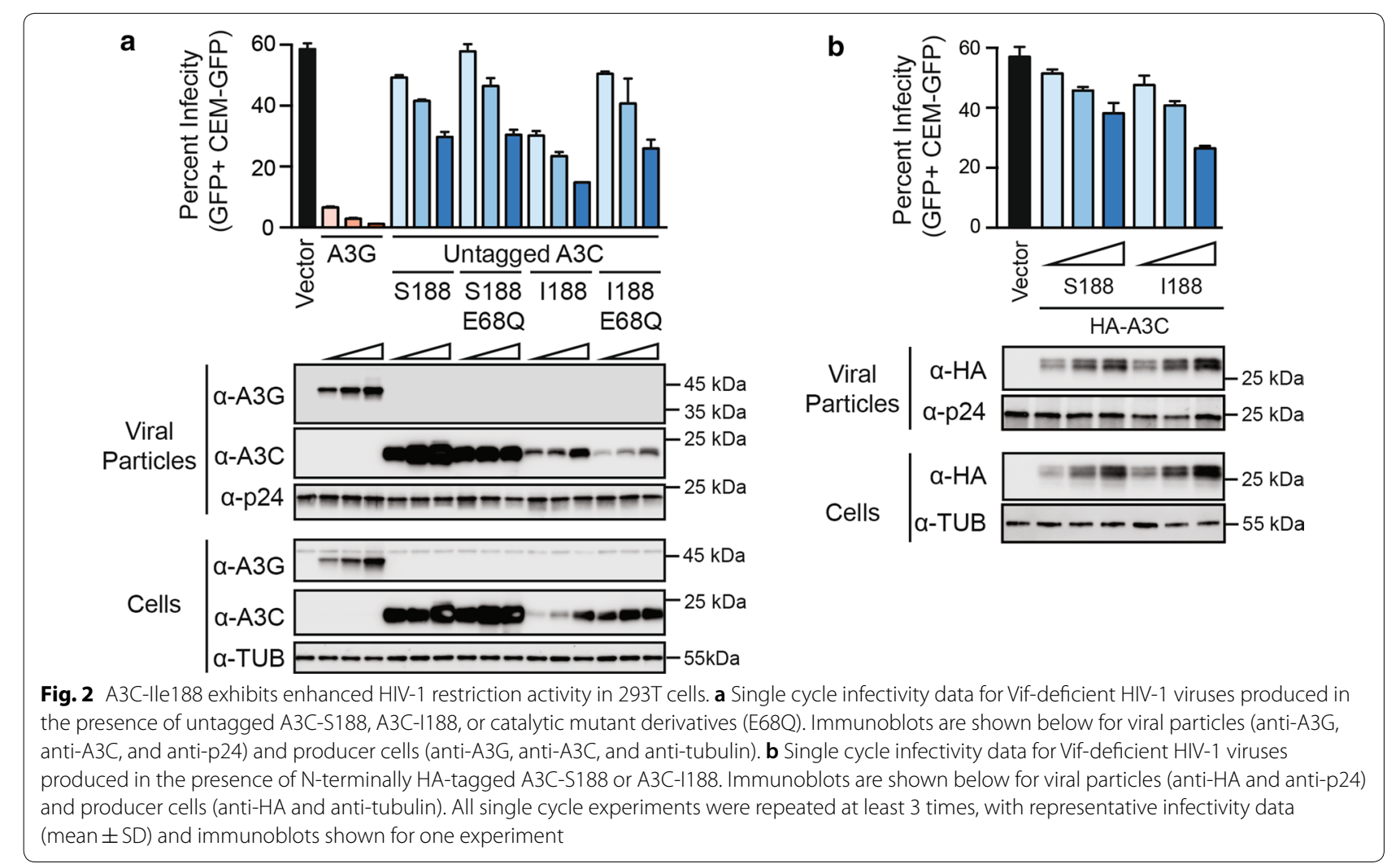
experiments is that the Ile188 enzyme is approximately twofold more restrictive than the Ser188 enzyme.

\section{A3C disruption and variant complementation has little effect on HIV-1 replication kinetics in non-permissive CEM2n cells}

Little is known about the role of endogenous $\mathrm{A} 3 \mathrm{C}$ in HIV-1 restriction in T cells. Endogenous A3C is detected readily in non-permissive $\mathrm{H} 9$ and CEM2n $\mathrm{T}$ cell lines, but not in the permissive SupT11 $\mathrm{T}$ cell line using the commercial anti-A3C antibody described above (Fig. 3a). Cas9/gRNA-mediated genome editing was used to disrupt endogenous $A 3 C$ in CEM2n [24]. The parental CEM2n line was transduced with a vector expressing Cas9 and an $A 3 C$-specific guide RNA, and limiting dilution was used to isolate clones harboring no detectable A3C protein by immunoblot (e.g., clone 1 in Fig. 3b). Genomic DNA sequencing of the gRNA-targeted exon 3 region of $A 3 C$ revealed multiple mutant alleles and, importantly, no wild-type sequences (Fig. 3c). The presence of 3 distinct alleles indicates that the original neardiploid cell line CEM2n [24] may have increased ploidy during culture or duplicated the $A 3 C$ region of the genome. Regardless, A3C-disrupted clones showed no overt phenotypes such as morphology or growth rates in comparison to non-targeted sister clones (data not shown).

Next, a complementation experiment was set-up by transducing the $A 3 C$ mutant clone described above with viruses expressing A3C-Ser188, A3C-Ile188, or an empty vector as a negative control. Each construct also expressed eGFP to control for transduction efficiency (and to help mitigate the fact that the untagged $\mathrm{A} 3 \mathrm{C}$ Ile188 variant cannot be detected with available antibodies, as shown in Fig. 1). In all instances, near complete transduction efficiency was confirmed by GFP+ flow cytometry (Fig. 3d). Moreover, immunoblotting showed that complemented A3C-Ser188 expression levels are similar to endogenous levels normally found in the parental CEM2n line (Fig. 3e). As explained above, protein-level expression of A3C-Ile188 could not be measured accurately due to Ser 188 likely being part of the main epitope recognized by the antibody. Nevertheless, these two variants are expected to express at similar levels because the vectors are otherwise isogenic, the Ser188 and Ile188 variants express similarly with $\mathrm{N}$-terminal tags, and the eGFP levels of the complemented pools are almost indistinguishable (and results below show a phenotype with the same A3C-Ile188 construct).

To address the role of endogenous A3C (Ser188/ Ser188) and to compare complemented A3C-Ser188 and A3C-Ile188 variants in parallel in HIV-1 spreading infections, transduced cell pools were infected with HIV-1 ${ }_{\text {IIIB }}$ or a Vif-deficient derivative and virus replication was monitored over time. Neither the Cas9/gRNA-mediated disruption of endogenous $\mathrm{A} 3 \mathrm{C}$ nor complementation with either natural $\mathrm{A} 3 \mathrm{C}$ variant altered the kinetics of Vif-proficient virus replication with all CEM2n $\triangle \mathrm{A} 3 \mathrm{C}$ derivatives showing peak infectivity at 7 days post-infection (Fig. 3f). In contrast, CEM2n $\triangle \mathrm{A} 3 \mathrm{C}$ derivatives remained fully non-permissive to Vif-deficient virus replication presumably due to expression of other endogenous restrictive A3 enzymes including A3D, A3F, A3G and $\mathrm{A} 3 \mathrm{H}[9,24]$ (Fig. 3g). Vif-deficient virus replication in the permissive SupT11 cell line confirmed infectivity of the viral stock (Fig. 3f, g). Taken together, these results demonstrate that $\mathrm{A} 3 \mathrm{C}$ disruption is not sufficient to render CEM2n cells detectably permissive to Vif-deficient HIV-1 replication, and that complementation with A3C-Ser188 or A3C-Ile188 is unable to alter the kinetics of virus replication in the presence of Vif, potentially because both variants are targeted for degradation by Vif at similar efficiencies [11].

\section{Stable expression of A3C-Ser188 and A3C-Ile188 in SupT11 cells restricts Vif-deficient HIV-1 replication and inflicts G-to-A mutations}

The phenotypically redundant antiviral activities of A3D, A3F, A3G, and A3H make it challenging to study $\mathrm{A} 3 \mathrm{C}$ activity in non-permissive $\mathrm{T}$ cell lines such as CEM2n. Therefore, permissive, non-A3 expressing T cell lines such as SupT11 provide robust systems for comparing the antiviral activities of single DNA deaminases and mutant derivatives [9, 20, 25-27]. Prior

\footnotetext{
(See figure on next page.)

Fig. 3 HIV-1 replication phenotypes following A3C disruption and variant complementation in non-permissive CEM2n cells. a Immunoblots of endogenous A3C in SupT11, H9, and CEM2n cells. Tubulin was used as a loading control. b Immunoblots of endogenous A3C in CEM2n clones following targeted disruption of $A 3 C$ exon 3 by Cas9/gRNA complexes. Tubulin was used as a loading control. c $A 3 C$ exon 3 sequences from parental CEM2n and an A3C-disrupted clone (CEM2n $\triangle A 3 C$ ). d Flow cytometry plots for CEM2n $\triangle A 3 C$ cell pools $72 \mathrm{~h}$ post-transduction with GFP-reporter complementation vectors. The percentage of GFP+ cells is indicated for each population. e Immunoblots of $A 3 C$ in the parental CEM $2 \mathrm{n}$ line, CEM2n $\triangle A 3 C$, and complemented CEM2n $\triangle A 3 C$ derivatives. Tubulin was used as a loading control. $\mathbf{f}, \mathbf{g}$ Spreading infection kinetics of Vif-proficient and Vif-deficient HIV-1 (initial $\mathrm{MOI}=0.02$ ) in CEM2n, CEM2n $\triangle A 3 C$, and the indicated complemented conditions. SupT11 cells are included as a control permissive cell type. Virus infectivity was determined by infection of CEM-GFP with culture supernatants followed $48 \mathrm{~h}$ later by quantification with flow cytometry. Each spreading infection experiment was performed 3 times and representative data are shown
} 


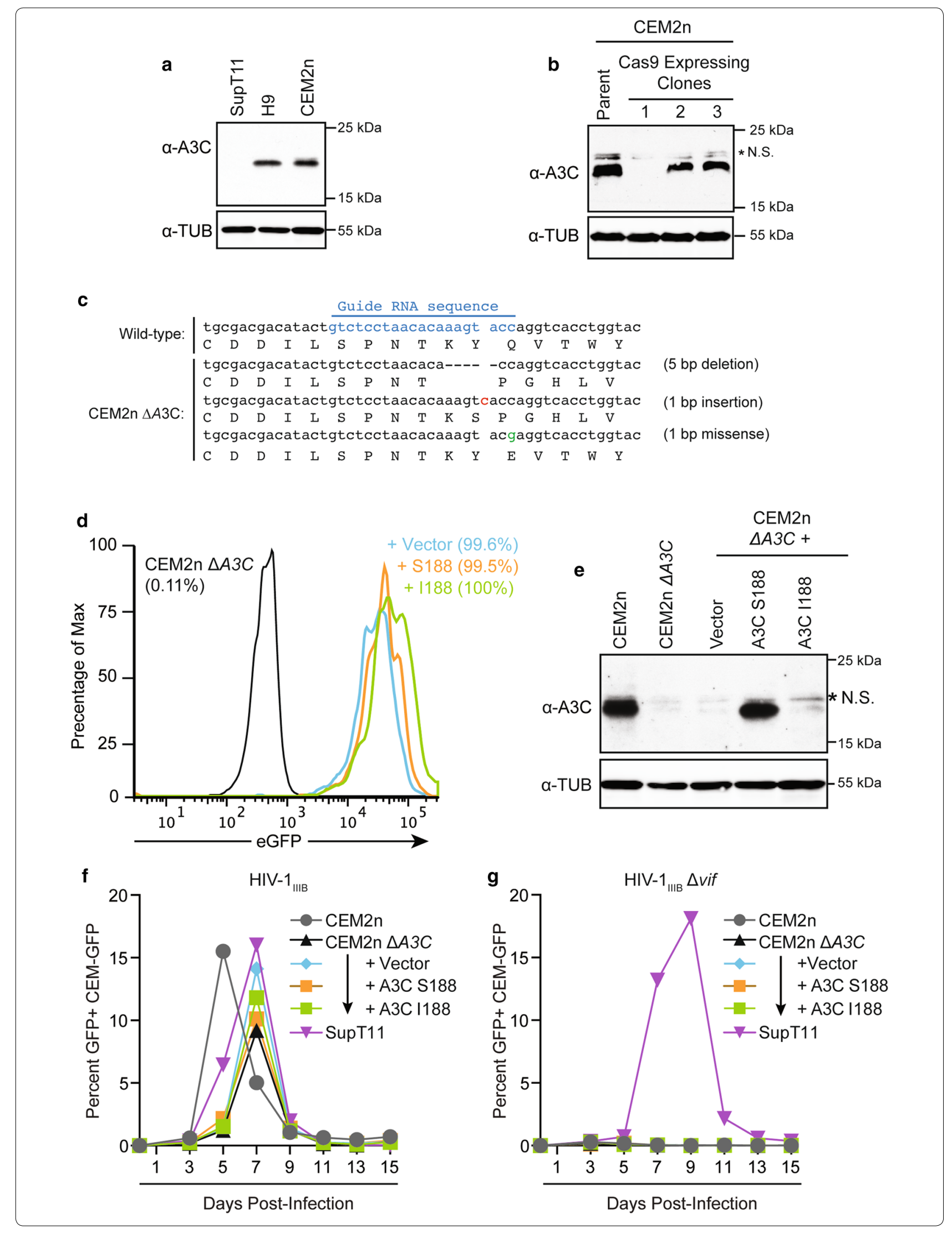


to knowledge of $\mathrm{A} 3 \mathrm{C}-\mathrm{Ile} 188$, we reported that $\mathrm{A} 3 \mathrm{C}$ Ser188 is unable to restrict Vif-deficient HIV-1 when expressed stably in this system [9]. These studies were additionally limited by the utilization of a C-terminal triple-HA epitope tag, which based on results above is detrimental to A3C expression (Fig. 1). Therefore, the intrinsic restriction activities of untagged A3C-Ser188 and A3C-Ile188 were compared following stable transduction and expression in SupT11 cells. As above, efficient transduction was confirmed by GFP+ flow cytometry for all constructs (Fig. 4a) and by immunoblotting for A3C-Ser188 (Fig. 4b).

The resulting SupT11 pools were infected with Vifdeficient HIV-1 $(\mathrm{MOI}=0.01)$ and virus replication kinetics were monitored over time. Both A3C-Ser188 and $\mathrm{A} 3 \mathrm{C}-\mathrm{Il} 188$ suppressed virus replication, but the latter variant imposed a reproducibly but not significantly greater delay (Fig. 4c). Moreover, in addition to imposing replication delays, the spreading infection peaks were lower than those in the vector control condition $(28 \pm 14,12 \pm 10$, and $9 \pm 6 \%$ GFP-positive reporter cells for vector, A3C-Ser188, and A3C-Ile188 conditions, respectively; $\mathrm{n}=4$ biologically independent experiments).

To ask whether $\mathrm{A} 3 \mathrm{C}$ inflicts G-to-A mutations in HIV-1 genomic sequences, high-fidelity PCR was used to recover proviral DNA from the HIV-1 infected SupT11 derivatives after 9 and 13 days of spreading infection. A minimum of 10 independent $564 \mathrm{bp}$ pol region DNA amplicons were cloned and sequenced for each condition. These analyses revealed that both $\mathrm{A} 3 \mathrm{C}$ variants caused significant increases in overall G-to-A mutation loads (twofold for Ser188 and fourfold for Ile188; Fig. 4d, e). In both instances, the underlying mutation spectra showed evidence for both $5^{\prime}$-GA-to-AA and 5'-GG-toAG mutations due to cDNA strand $5^{\prime}$-TC-to-TU and $5^{\prime}$-CC-to-CU deamination events, respectively (Fig. 4e). These proviral DNA sequencing analyses showed that both A3C-Ser188 and A3C-Ile188 have the capacity to inflict G-to-A mutations in viral DNA during a spreading infection in a $\mathrm{T}$ cell model system. However, in direct comparison to A3G, much lower frequencies of G-to-A mutation are observed suggesting some of the antiviral activity of $\mathrm{A} 3 \mathrm{C}$ may also be due to a deamination-independent mechanism (supported by data in Fig. 2a).

\section{Discussion}

The A3 DNA cytosine deaminase family imposes a significant barrier to productive HIV-1 infection, especially for viruses lacking Vif. Prior studies converged on A3D, A3F, $\mathrm{A} 3 \mathrm{G}$ and $\mathrm{A} 3 \mathrm{H}$ as the $\mathrm{HIV}-1$ restrictive $\mathrm{A} 3$ repertoire in CD4+ T cells (e.g., [9, 20, 28, 29]; reviewed by [2, 4, 30]). Our current studies indicate that A3C-Ile188 has the potential to contribute to HIV-1 restriction in human T cells. Our studies confirm prior 293T-based single cycle results $[11,23]$ and, importantly, extend this conclusion to a relevant model T cell line SupT11. Importantly, our studies show that both the common (Ser188) and the rare (Ile188) A3C variants are capable of inflicting G-to-A mutations during spreading infection in $\mathrm{T}$ lymphocytes with the rare variant inflicting a twofold higher mutation frequency. This increase in G-to-A mutations may be explained by an increased capacity to dimerize and to function in a processive manner [23]. This result raises the possibility that both variants may contribute to HIV-1 diversity. However, additional work will be needed to extend these findings to primary $\mathrm{T}$ lymphocytes, for instance, through an analysis of endogenous $\mathrm{A} 3 \mathrm{C}$ variants following Cas9/gRNA-mediated deletion of A3D/F/ $\mathrm{G} / \mathrm{H}$ ex vivo. Such studies would also be facilitated by an anti-A3C monoclonal antibody (yet to be developed to our knowledge) that binds equally well to both naturally occurring A3C-Ser188 and A3C-Ile188 variants.

The seven gene human $A 3$ locus is variable in the human population, consistent with an overall function of the encoded enzymes in innate immunity and previously documented evidence for positive selection [11, 31-34]. Known variations include complete inactivation of $A 3$ family members (29.5 kb $A 3 B$ deletion [16] and $\mathrm{A} 3 \mathrm{H}-$ $\triangle$ Asn15 [18]), hypomorphic alleles (A3H-G105 [18]), and hypermorphic alleles such as the A3C-Ile188 variant (prior work [11, 23] and this study). Several other coding and non-coding variants have been documented but, thus far, their functional relevance is less clear.

$\mathrm{A} 3 \mathrm{C}$ is conserved in primates and phylogenetic comparisons show evidence for positive selection, including

\footnotetext{
(See figure on next page.)

Fig. 4 Stable Expression of both A3C-Ile188 and A3C-Ser188 in SupT11 cells provides a partial block to Vif-deficient HIV-1 replication. a Flow cytometry plots for SupT11 cells $72 \mathrm{~h}$ after transduction with the indicated GFP-marked complementation vectors. The percentage of GFP+ cells is indicated for each condition. $\mathbf{b}$ Immunoblots of A3C in SupT11 cells transduced with constructs expressing A3C-Ser188, A3C-lle188, or an empty control vector. Tubulin was used as a loading control. c Representative spreading infection data for Vif-deficient HIV-1 (MOI =0.01) in SupT11 cells expressing A3C-Ser188, A3C-Ile188, or an empty control vector. Virus infectivity was determined by infection of CEM-GFP with culture supernatants followed $48 \mathrm{~h}$ later by quantification with flow cytometry. These data are from one of four biologically independent experiments. $\mathbf{d}$ Average G-to-A mutation loads for each condition. Error bars show \pm SD of 4 independent experiments. Statistical comparisons were done using Student's $t$ test. $p$ values above each panel are in comparison to vector control or A3C-Ser188. e Mutation data for each condition
} 


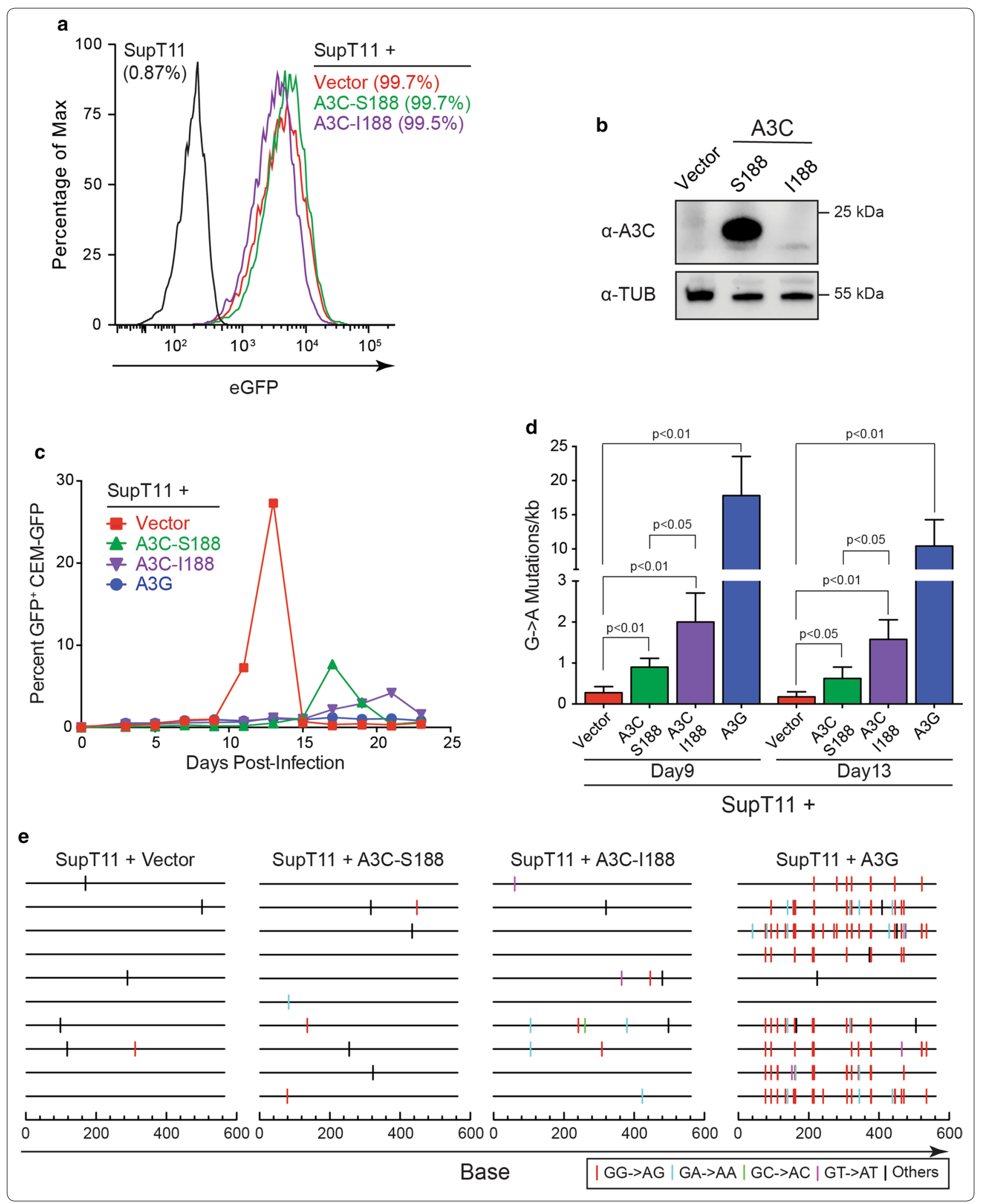


multiple amino acid substitutions within the predicted Vif-binding interface $[11,13,35]$. This suggests an ongoing conflict between ancestral lentiviruses with Vif and ancestral $\mathrm{A} 3 \mathrm{C}$ enzymes. Consistent with this idea, cross species comparisons have shown restriction of SIVagm by human $\mathrm{A} 3 \mathrm{C}[11,14]$. Taken together with the fact that HIV-1 Vif still degrades A3C and HIV-1 infection induces $A 3 C$ expression [9, 14, 35-37], it is possible that A3C-Ser188 and A3C-Ile188 still impose some (albeit low) selective pressure on present day HIV-1 (or SIV in recent evolutionary history) [9]. Alternatively, A3D and/ or $\mathrm{A} 3 \mathrm{~F}$ are still so similar to $\mathrm{A} 3 \mathrm{C}$ that molecular mimicry within the Vif binding surface may be sufficient to maintain Vif function against A3C.

A3C amino acid 188 is located in the C-terminal helix, which is physically separated from the Vif binding interface [13]. However, phylogenetic comparisons for this region are interesting. Within the human A3 repertoire, most enzymes have Ile at the analogous structural position suggesting a need for greater restriction activity [11]. Sequence comparisons across divergent primate species indicate that Ile188 is the ancestral primate residue, occurring in divergent primate species including the orangutan, gibbon, siamang, and all sequenced Old World monkeys [11]. In contrast, Ser188 is found in humans and other hominids including chimpanzees, gorillas, and bonobos. Curiously, chimpanzee and gorilla A3C proteins have a HIV- 1 restriction activity that is comparable to human A3C-Ile188, despite harboring Ser at the analogous amino acid position, suggesting that additional amino acid differences between hominid species may play a role in defining intrinsic restriction activity [23]. Biochemical studies of these enzymes have revealed a correlation between self-dimerization of A3C in solution and HIV-1 restriction activity (observed for human A3C-Ile188, chimp A3C, and gorilla A3C, but not human A3C-Ser188) [23]. The biochemical characterization of additional primate $\mathrm{A} 3 \mathrm{C}$ proteins will be needed to test the broad relevance of dimerization in $\mathrm{A} 3 \mathrm{C}$-dependent restriction of primate lentiviruses, though these findings strongly suggest that Ile 188 is not the only determinant for enhanced restriction activity.

The overall contribution of A3C-Ile188 to HIV-1 replication in vivo remains unclear at this time, though relative comparisons in single cycle infection experiments suggest that its antiviral activity will pale in comparison to the potent restriction activity of A3G (e.g., Fig. 2a). Nevertheless, studies here and recent reports [11, 23] on A3C combine to reveal an additional example of genetic variation within the human A3 repertoire that may influence HIV-1 evolvability and pathogenesis in the primary cellular reservoir, CD4+ T cells. Future studies leveraging large patient cohorts of known $A 3 C$ genotypes, with due attention to other variations within the locus, may be able to address this possibility.

\section{Conclusions}

We show that the rare human A3C-Ile188 variant exhibits enhanced restriction activity against Vif-deficient HIV-1 in a 293T-based single cycle infection system, providing independent verification of prior work [11, 23]. Using Cas9-mediated gene disruption in non-permissive CEM2n T cells, we found that endogenous A3C is not required for Vif-deficient HIV-1 restriction, most likely due to the dominant restriction activities of $\mathrm{A} 3 \mathrm{D}$, A3F, A3G and A3H. Complementation of A3C-disrupted CEM2n cells also had no effect on virus replication kinetics. However, stable expression of A3C-Ile188 can convert the normally permissive SupT $11 \mathrm{~T}$ cells to a less permissive phenotype with respect to Vif-deficient virus replication. Vif-deficient viruses also accumulate G-to-A mutations from A3C-Ile188, as well as from A3C-Ser188, under these spreading infection conditions. Taken together, these findings support a role for A3C in HIV-1 restriction and G-to-A mutation in $\mathrm{T}$ cells, and highlight an additional level of variation within the human $A 3$ gene locus that may effect HIV-1 adaptation and pathogenesis in vivo.

\section{Methods \\ Cell lines}

SupT11 [9], SupT11-A3G [38], CEM2n [24] and derivative cell lines were maintained in RPMI (Hyclone) supplemented with 10\% FBS (Thermo Fisher) and 1\% penicillin/streptomycin. 293T cells were maintained in DMEM (Hyclone) supplemented with 10\% FBS (Gibco) and $1 \%$ penicillin/streptomycin. $293 \mathrm{~T}$ cells were transfected with TransIT LTI (Mirus) according to the manufacture's protocol. All experiments were harvested $48 \mathrm{~h}$ post-transfection.

\section{A3C expression plasmids}

The C-terminally triple-HA tagged $\mathrm{A} 3 \mathrm{C}$ construct in the pcDNA3.1 $(+)$ backbone has been described [9]. The $\mathrm{N}$-terminally triple-HA tagged $\mathrm{A} 3 \mathrm{C}$ constructs were ligated into the pcDNA3.1(+) backbone with a triple-HA epitope sequence between the NheI and HindIII cloning sites. Untagged A3C constructs were assembled similarly into an epitope tag-free pcDNA3.1(+) backbone. Untagged A3C variants were subcloned into the CSU6IDR2 IRES-eGFP lentiviral expression vector (previously described [10] but modified here to express eGFP instead of dsRed) for stable transduction experiments in CEM2n and SupT11 cells. 


\section{Immunoblots}

Cell pellets were lysed directly in $2.5 x$ Laemmli sample buffer, separated by $12.5 \%$ SDS-PAGE, and transferred to PVDF-FL membranes (Millipore). Membranes were blocked in $4 \%$ milk in PBS and incubated with primary antibodies diluted in 4\% milk in PBS supplemented with $0.1 \%$ Tween20. Secondary antibodies were diluted in $4 \%$ milk in PBS supplemented with $0.1 \%$ Tween20 and $0.01 \%$ SDS. Membranes were imaged on a LI-COR Odyssey instrument. Primary antibodies used in these studies were rabbit anti-A3C (Proteintech 10591-1-AP), rabbit anti-A3G (NIH AIDS Reagent Program 10201 courtesy of J. Lingappa), rabbit anti-HA (Cell signaling C29F4), mouse anti-Tubulin (Sigma T5168), mouse anti-HIV-1 p24/CA (NIH AIDS Reagent Program 3537 courtesy of B. Chesebro and K. Wehrly), and mouse anti-HIV-1 Vif (NIH AIDS Reagent Program 6459 courtesy of M. Malim). Secondary antibodies employed were IRdye $800 \mathrm{CW}$ goat anti-rabbit (LI-COR 827-08365) and Alexa Fluor 680 goat anti-mouse (Molecular Probes A-21057), except when detecting the anti-A3C antibody, which was probed with an anti-Rb HRP-conjugated secondary antibody (Bio Rad 1706515) and visualized using Super Signal West Femto Maximum Sensitivity Substrate (ThermoFisher Scientific). The following A3C antibodies failed to detect overexpressed $\mathrm{N}$-terminally tagged $\mathrm{A} 3 \mathrm{C}$ variants by immunoblotting: rabbit anti-A3C (Abcam ab181356), rabbit anti-A3C (Abcam ab209560), rabbit anti-A3C (LSBio LS-B14538), and mouse anti-A3C (Sigma SAB1403204).

\section{HIV-1 single-cycle infectivity experiments}

293T-based HIV-1 single cycle infectivity assays were performed by co-transfecting 293T cells with an HIV$1_{\text {IIIB }}$ A200C vif-deficient molecular clone derivative (pIIIB $\Delta v i f$ ) along with the indicated APOBEC3 expression construct or an empty vector control $[39,40] .48 \mathrm{~h}$ later, cell free supernatants containing progeny viruses were used to infect CEM-GFP reporter cells. Reporter cell infectivity was assayed $48 \mathrm{~h}$ post infection by flow cytometry using a Becton Dickinson FacsCanto II instrument.

\section{A3C disruption by Cas9/gRNA}

A gRNA sequence homologous to $5^{\prime}$-GGG GCT CCG CAG CCT GAG TC- $3^{\prime}$ in $A 3 C$ exon 3 was generated using the CRISPR Design Tool (Massachusetts Institute of Technology) and cloned into the lentiCRISPRv1 vector (Addgene \#49535) according to the accompanying Zhang lab protocol [41]. This targeting construct was generated by annealing oligos 5'-ACA CCG TCT CCT AAC ACA AAG TAC CG-3' and 5'-AAA ACG GTA CTT TGT GTT AGG AGA CG-3' and ligating the resulting dsDNA fragment into BsmBI-digested lentiCRISPRv1. 293T cells were transfected with the lentiCRISPRv1 targeting construct along with $\mathrm{p} \Delta$-NRF (HIV-1 gag, pol, rev, tat genes) and pMDG (VSV-G) expression vectors and, $48 \mathrm{~h}$ later, virus-containing supernatants were filtered $(0.45 \mu \mathrm{m})$ and concentrated by centrifugation $(22,000 \times g, 2 \mathrm{~h}$, $\left.10{ }^{\circ} \mathrm{C}\right)$. Viral pellets were resuspended in complete RPMI and incubated with cells for $48 \mathrm{~h}$ before being placed under drug selection ( $1 \mu \mathrm{g} / \mathrm{mL}$ puromycin). Clones were isolated by limiting dilution of the drug resistant cell pool, and $\mathrm{A} 3 \mathrm{C}$ expression was analyzed by immunoblotting lysates from individual clones.

\section{A3C complementation}

CSU6-IDR2 IRES-eGFP vectors encoding untagged and $\mathrm{N}$-terminally HA-tagged A3C variants were co-transfected into $293 \mathrm{~T}$ cells with $\mathrm{p} \Delta$-NRF (HIV-1 gag-pol-revtat) and pMDG (VSV-G). $48 \mathrm{~h}$ post transfection, culture supernatants were filtered $(0.45 \mu \mathrm{m})$ and concentrated by centrifugation $\left(22,000 \times g, 2 \mathrm{~h}, 10{ }^{\circ} \mathrm{C}\right)$. Viral pellets were resuspended in complete RPMI and used to directly infect SupT11 and CEM2n target cells. Transduced cell populations were assayed for GFP expression by flow cytometry and $\mathrm{A} 3 \mathrm{C}$ expression was measured by immunoblotting $72 \mathrm{~h}$ post-transduction.

\section{HIV-1 spreading infections}

HIV-1 spreading infections were performed as described [9]. Briefly, replication-competent HIV-1 stocks were produced by transfecting $293 \mathrm{~T}$ cells with HIV-1 $1_{\text {III }}$ A200C wild-type (pIIIB) or vif-deficient (pIIIB $\Delta v i f$ ) molecular clone derivatives $[39,40] .48 \mathrm{~h}$ post-transfection, culture supernatants were filtered $(0.45 \mu \mathrm{m})$, and viral titers were determined by serial titration on CEMGFP reporter cells. CEM2n and SupT11 derivative cell lines were infected accordingly, and virus replication was monitored every other day (beginning on day 3 postinfection) by infecting CEM-GFP reporter cells with culture supernatants. Infected CEM-GFP cells were fixed at $48 \mathrm{~h}$ post-infection and assayed for GFP expression by flow cytometry.

\section{Proviral DNA G-to-A mutation analyses}

SupT11 cells at day 9 and 13 of spreading infections were harvested and genomic DNA (including proviral DNA) was prepared (Puregene) for sequencing analysis as described [42]. The viral pol region was amplified by nested PCR with RSH4196/4197 (5'-TCC ART ATT TRC CAT AAA RAA AAA- $3^{\prime}$ and $5^{\prime}$-TTY AGA TTT TTA AAT GGY TYT TGA-3') as outer primers (876 bp) and RSH4205/4206 (5'-AAT ATT CCA RTR TAR CAT RAC AAA AAT- $3^{\prime}$ and $5^{\prime}$-AAT GGY TYT TGA TAA ATT TGA TAT GT- $3^{\prime}$ ) as inner primers $(564 \mathrm{bp}$ ) and then the amplified products were subjected to pJET cloning and 
Sanger DNA sequencing (GENEWIZ). Sequences were aligned using Sequencher (Gene Codes Corporation) and analyzed for hypermutation by the Los Alamos Hypermut algorithm [43].

\section{Authors' contributions}

BDA, TI, RSH: conceived and designed the experiments; BDA, TI, SAM: Performed the experiments; BDA, TI, SAM, RSH: Analyzed the data; BDA, TI, AS WLB: Contributed key reagents; RSH: Wrote the paper. All authors read and approved the final manuscript.

\begin{abstract}
Author details
${ }^{1}$ Department of Biochemistry, Molecular Biology and Biophysics, Masonic Cancer Center, Center for Genome Engineering, Institute for Molecular Virology, University of Minnesota, Minneapolis, MN, USA. ${ }^{2}$ Howard Hughes Medical Institute, University of Minnesota, 2231 6th St. S.E., Minneapolis, MN 55455, USA.
\end{abstract}

\section{Acknowledgements}

We thank Christopher Richards, Nadine Shaban, Gabriel Starrett, and Diako Ebrahimi for helpful comments throughout this study. R.S.H. is the Margaret Harvey Schering Land Grant Chair for Cancer Research, a Distinguished McKnight University Professor, and an Investigator of the Howard Hughes Medical Institute.

\section{Competing interests}

$\mathrm{RSH}$ is a co-founder, shareholder, and consultant of ApoGen Biotechnologies Inc. The other authors declare no competing interests.

\section{Availability of data and materials}

The datasets and original reagents used and/or analyzed as part of this study are available from the corresponding author on reasonable request.

\section{Consent for publication}

Not applicable.

Ethics approval and consent to participate

Not applicable.

\section{Funding}

This work was supported by NIAID R37 Al064046 and NCI R21 CA206309. BDA received salary support from NIAID F31 Al116305, and ASM from NSF GRFP 00039202.

\section{Publisher's Note}

Springer Nature remains neutral with regard to jurisdictional claims in published maps and institutional affiliations.

Received: 15 November 2018 Accepted: 6 December 2018 Published online: 17 December 2018

\section{References}

1. Altfeld M, Gale M Jr. Innate immunity against HIV-1 infection. Nat Immunol. 2015;16:554-62.

2. Simon V, Bloch N, Landau NR. Intrinsic host restrictions to HIV-1 and mechanisms of viral escape. Nat Immunol. 2015;16:546-53.

3. Harris RS, Hultquist JF, Evans DT. The restriction factors of human immunodeficiency virus. J Biol Chem. 2012;287:40875-83.

4. Harris RS, Dudley JP. APOBECs and virus restriction. Virology. 2015:479-480C:131-45

5. Stavrou S, Ross SR. APOBEC3 proteins in viral immunity. J Immunol. 2015;195:4565-70.

6. Yu Y, Xiao Z, Ehrlich ES, Yu X, Yu XF. Selective assembly of HIV-1 Vif-Cul5ElonginB-ElonginC E3 ubiquitin ligase complex through a novel SOCS box and upstream cysteines. Genes Dev. 2004;18:2867-72.
7. Jäger S, Kim DY, Hultquist JF, Shindo K, LaRue RS, Kwon E, Li M, Anderson BD, Yen L, Stanley D, et al. Vif hijacks CBF-beta to degrade APOBEC3G and promote HIV-1 infection. Nature. 2011:481:371-5.

8. Zhang W, Du J, Evans SL, Yu Y, Yu XF. T-cell differentiation factor CBFbeta regulates HIV-1 Vif-mediated evasion of host restriction. Nature. 2011;481:376-9.

9. Hultquist JF, Lengyel JA, Refsland EW, LaRue RS, Lackey L, Brown WL Harris RS. Human and rhesus APOBEC3D, APOBEC3F, APOBEC3G, and APOBEC $3 \mathrm{H}$ demonstrate a conserved capacity to restrict Vif-deficient HIV-1. J Virol. 2011;85:11220-34.

10. Anderson BD, Harris RS. Transcriptional regulation of APOBEC3 antiviral immunity through the CBF-beta/RUNX axis. Sci Adv. 2015;1:e1500296.

11. Wittkopp CJ, Adolph MB, Wu LI, Chelico L, Emerman M. A single nucleotide polymorphism in human APOBEC3C enhances restriction of lentiviruses. PLoS Pathog. 2016;12:e1005865.

12. Zhang Z, Gu Q, Jaguva Vasudevan AA, Jeyaraj M, Schmidt S, Zielonka J, Perkovic M, Heckel JO, Cichutek K, Haussinger D, et al. Vif proteins from diverse human immunodeficiency virus/simian immunodeficiency virus lineages have distinct binding sites in A3C. J Virol. 2016;90:10193-208.

13. Kitamura S, Ode H, Nakashima M, Imahashi M, Naganawa Y, Kurosawa T, Yokomaku Y, Yamane T, Watanabe N, Suzuki A, et al. The APOBEC3C crystal structure and the interface for HIV-1 Vif binding. Nat Struct Mol Biol. 2012;19:1005-10.

14. Yu Q, Chen D, Konig R, Mariani R, Unutmaz D, Landau NR. APOBEC3B and $A P O B E C 3 C$ are potent inhibitors of simian immunodeficiency virus replication. J Biol Chem. 2004;279:53379-86

15. Duggal NK, Fu W, Akey JM, Emerman M. Identification and antiviral activity of common polymorphisms in the APOBEC3 locus in human populations. Virology. 2013;443:329-37.

16. Kidd JM, Newman TL, Tuzun E, Kaul R, Eichler EE. Population stratification of a common APOBEC gene deletion polymorphism. PLoS Genet. 2007;3:e63.

17. Starrett GJ, Luengas EM, McCann JL, Ebrahimi D, Temiz NA, Love RP, Feng Y, Adolph MB, Chelico L, Law EK, et al. The DNA cytosine deaminase APOBEC3H haplotype I likely contributes to breast and lung cancer mutagenesis. Nat Commun. 2016;7:12918.

18. OhAinle M, Kerns JA, Li MM, Malik HS, Emerman M. Antiretroelement activity of APOBEC3H was lost twice in recent human evolution. Cell Host Microbe. 2008:4:249-59.

19. Wang X, Abudu A, Son S, Dang Y, Venta PJ, Zheng YH. Analysis of human APOBEC3H haplotypes and anti-human immunodeficiency virus type 1 activity. J Virol. 2011;85:3142-52.

20. Refsland EW, Hultquist JF, Luengas EM, Ikeda T, Shaban NM, Law EK, Brown WL, Reilly C, Emerman M, Harris RS. Natural polymorphisms in human APOBEC $3 \mathrm{H}$ and HIV-1 Vif combine in primary T lymphocytes to affect viral G-to-A mutation levels and infectivity. PLoS Genet. 2014;10:e1004761.

21. Ebrahimi D, Richards CM, Carpenter MA, Wang J, Ikeda T, Becker JT, Cheng AZ, McCann JL, Shaban NM, Salamango DJ, et al. Genetic and mechanistic basis for APOBEC3H alternative splicing, retrovirus restriction, and counteraction by HIV-1 protease. Nat Commun. 2018;9:4137.

22. Dang $Y$, Siew LM, Wang $X$, Han $Y$, Lampen R, Zheng YH. Human cytidine deaminase APOBEC3H restricts HIV-1 replication. J Biol Chem. 2008;283:11606-14

23. Adolph MB, Ara A, Feng Y, Wittkopp CJ, Emerman M, Fraser JS, Chelico L. Cytidine deaminase efficiency of the lentiviral viral restriction factor APOBEC3C correlates with dimerization. Nucleic Acids Res. 2017:45:3378-94

24. Refsland EW, Hultquist JF, Harris RS. Endogenous origins of HIV-1 G-to-A hypermutation and restriction in the nonpermissive T cell line CEM2n. PLoS Pathog. 2012;8:e1002800.

25. Ooms M, Brayton B, Letko M, Maio SM, Pilcher CD, Hecht FM, Barbour JD, Simon V. HIV-1 Vif adaptation to human APOBEC3H haplotypes. Cell Host Microbe. 2013;14:411-21.

26. Letko M, Booiman T, Kootstra N, Simon V, Ooms M. Identification of the HIV-1 Vif and human APOBEC3G protein interface. Cell Rep. 2015;13:1789-99.

27. Ooms M, Letko M, Simon V. The structural interface between HIV-1 Vif and human APOBEC3H. J Virol. 2017;91:e02289. 
28. Nakano Y, Misawa N, Juarez-Fernandez G, Moriwaki M, Nakaoka S, Funo T, Yamada E, Soper A, Yoshikawa R, Ebrahimi D, et al. HIV-1 competition experiments in humanized mice show that APOBEC3H imposes selective pressure and promotes virus adaptation. PLoS Pathog. 2017;13:e1006348.

29. Krisko JF, Martinez-Torres F, Foster JL, Garcia JV. HIV restriction by APOBEC3 in humanized mice. PLoS Pathog. 2013;9:e1003242.

30. Malim MH, Bieniasz PD. HIV restriction factors and mechanisms of evasion. Cold Spring Harb Perspect Med. 2012;2:a006940.

31. McLaughlin RN Jr, Gable JT, Wittkopp CJ, Emerman M, Malik HS. Conservation and innovation of $A P O B E C 3 A$ restriction functions during primate evolution. Mol Biol Evol. 2016;33:1889-901.

32. Duggal NK, Malik HS, Emerman M. The breadth of antiviral activity of APOBEC3DE in chimpanzees has been driven by positive selection. J Virol. 2011:85:11361-71.

33. Sawyer SL, Emerman M, Malik HS. Ancient adaptive evolution of the primate antiviral DNA-editing enzyme APOBEC3G. PLoS Biol. 2004;2:E275.

34. OhAinle M, Kerns JA, Malik HS, Emerman M. Adaptive evolution and antiviral activity of the conserved mammalian cytidine deaminase APOBEC3H. J Virol. 2006;80:3853-62.

35. Smith $J L$, Pathak VK. Identification of specific determinants of human APOBEC3F, APOBEC3C, and APOBEC3DE and African green monkey APOBEC3F that interact with HIV-1 Vif. J Virol. 2010;84:12599-608.

36. Zhang W, Chen G, Niewiadomska AM, Xu R, Yu XF. Distinct determinants in HIV-1 Vif and human APOBEC3 proteins are required for the suppression of diverse host anti-viral proteins. PLoS ONE. 2008;3:e3963.
37. Refsland EW, Stenglein MD, Shindo K, Albin JS, Brown WL, Harris RS. Quantitative profiling of the full APOBEC3 mRNA repertoire in lymphocytes and tissues: implications for HIV-1 restriction. Nucleic Acids Res. 2010;38:4274-84.

38. Albin JS, Brown WL, Harris RS. Catalytic activity of APOBEC3F is required for efficient restriction of Vif-deficient human immunodeficiency virus. Virology. 2014;450-451:49-54.

39. Haché G, Shindo K, Albin JS, Harris RS. Evolution of HIV-1 isolates that use a novel Vif-independent mechanism to resist restriction by human APOBEC3G. Curr Biol. 2008;18:819-24.

40. Haché G, Abbink TE, Berkhout B, Harris RS. Optimal translation initiation enables Vif-deficient human immunodeficiency virus type 1 to escape restriction by APOBEC3G. J Virol. 2009;83:5956-60.

41. Sanjana NE, Shalem O, Zhang F. Improved vectors and genome-wide libraries for CRISPR screening. Nat Methods. 2014;11:783-4.

42. Ikeda T, Symeonides M, Albin JS, Li M, Thali M, Harris RS. HIV-1 adaptation studies reveal a novel Env-mediated homeostasis mechanism for evading lethal hypermutation by APOBEC3G. PLoS Pathog. 2018;14:e1007010.

43. Rose PP, Korber BT. Detecting hypermutations in viral sequences with an emphasis on $\mathrm{G} \rightarrow$ A hypermutation. Bioinformatics. 2000;16:400-1.
Ready to submit your research? Choose BMC and benefit from:

- fast, convenient online submission

- thorough peer review by experienced researchers in your field

- rapid publication on acceptance

- support for research data, including large and complex data types

- gold Open Access which fosters wider collaboration and increased citations

- maximum visibility for your research: over 100M website views per year

At BMC, research is always in progress.

Learn more biomedcentral.com/submissions 